\title{
A Two-Stage Offline-to-Online Multiobjective Optimization Strategy for Ship Integrated Energy System Economical/ Environmental Scheduling Problem
}

\author{
Qing An $\left(\mathbb{D},{ }^{1}\right.$ Jun Zhang $\left(\mathbb{D},{ }^{2}\right.$ Xin Li $\left(\mathbb{D},{ }^{3,4}\right.$ Xiaobing Mao $\mathbb{D}^{3},{ }^{3}$ Yulong Feng, ${ }^{5}$ Xiao Li, ${ }^{5}$ \\ Xiaodi Zhang $\mathbb{C}^{6}{ }^{6}$ Ruoli Tang, ${ }^{3,4}$ and Hongfeng Su $\mathbb{1}^{7}$ \\ ${ }^{1}$ Artificial Intelligence School, Wuchang University of Technology, Wuhan 430223, China \\ ${ }^{2}$ Zhejiang Electronic Information Products Inspection and Research Institute (Key Laboratory of Information \\ Security of Zhejiang Province), No. 50 Tian Mu Shan Road, Hangzhou, China \\ ${ }^{3}$ School of Energy and Power Engineering, Wuhan University of Technology, Wuhan 430063, China \\ ${ }^{4}$ Key Lab. of Marine Power Engineering and Tech. Authorized by MOT, Wuhan 430063, China \\ ${ }^{5}$ Shanghai Marine Diesel Engine Research Inistute, Shanghai, China \\ ${ }^{6}$ State Grid Beijing Electric Maintenance Company, Beijing 100080, China \\ ${ }^{7}$ Sichuan Vocational and Technical College of Communications, Chengdu 611130, China
}

Correspondence should be addressed to Jun Zhang; zj@zdjy.org.cn, Xin Li; xinli0503@hotmail.com, Xiaobing Mao; maoxiaobing2009@163.com, and Hongfeng Su; 568120525@qq.com

Received 3 December 2020; Revised 5 February 2021; Accepted 5 March 2021; Published 17 March 2021

Academic Editor: Chen Wang

Copyright ( 2021 Qing An et al. This is an open access article distributed under the Creative Commons Attribution License, which permits unrestricted use, distribution, and reproduction in any medium, provided the original work is properly cited.

\begin{abstract}
The economical/environmental scheduling problem (EESP) of the ship integrated energy system (SIES) has high computational complexity, which includes more than one optimization objective, various types of constraints, and frequently fluctuated load demand. Therefore, the intelligent scheduling strategies cannot be applied to the ship energy management system (SEMS) online, which has limited computing power and storage space. Aiming at realizing green computing on SEMS, in this paper a typical SIES-EESP optimization model is built, considering the form of decision vectors, the economical/environmental optimization objectives, and various types of real-world constraints of the SIES. Based on the complexity of SIES-EESPs, a two-stage offline-to-online multiobjective optimization strategy for SIES-EESP is proposed, which transfers part of the energy dispatch online computing task to the offline high-performance computer systems. The specific constraints handling methods are designed to reduce both continuous and discrete constraints violations of SIESEESPs. Then, an establishment method of energy scheduling scheme-base is proposed. By using the big data offline, the economical/environmental scheduling solutions of a typical year can be obtained and stored with more computing resources and operation time on land. Thereafter, a short-term multiobjective offline-to-online optimization approach by SEMS is considered, with the application of multiobjective evolutionary algorithm (MOEA) and typical schemes corresponding to the actual SIES-EESPs. Simulation results show that the proposed strategy can obtain enough feasible Pareto solutions in a shorter time and get well-distributed Pareto sets with better convergence performance, which can well adapt to the features of real-world SIES-EESPs and save plenty of operation time and storage space for the SEMS.
\end{abstract}

\section{Introduction}

With the increasing depletion of traditional fossil energy and the exhaust gas generated by ship combustion, the fuel consumption and emission pollution of marine diesel engine have become the main factors affecting the economy and environmental protection of ships [1-3]. In addition, due to the poor working conditions of the diesel engine, especially in the motor operating conditions, parts are easy to be damaged, and the daily maintenance cost is also the main economic factor. With the rapid development of world trade, ship transportation accounts for a considerable proportion, and $\mathrm{CO}_{2}$ and other 
exhaust gas produced by the shipping industry cannot be ignored [4]. Currently, there are two main forms of power generation on ships: traditional diesel generator and clean energy. Although the traditional diesel generator can provide strong power for ships, environmental pollution cannot be avoided. Because of its green and renewable nature, clean energy has a good development prospect in the future shipping market. Clean energy, including wind energy, solar energy, nuclear energy, fuel cells, and tidal energy, has been preliminarily applied on ships [5]. A multienergy ship can be defined as a ship with two or more kinds of energy storage equipment, energy supply unit, or energy conversion unit as the power source, and at least one of them can provide electric energy [6]. Compared with the pure electric ship, the multienergy ship has better endurance and redundancy and has the advantages of less fuel consumption, less pollution, and lower noise compared with the ship only using diesel generator set as power source $[7,8]$.

With the improvement of ship power grid capacity, it is necessary to improve the economy and environmental protection while meeting the reliability and safety of the ship integrated energy system (SIES). In other words, how to improve the computing performance of ship energy management system (SEMS) has become a significant issue. In the existing research, the EMS control strategy aiming at improving the safety, reliability, and fuel economy of ships mainly considers the following aspects: generator set limitation, load limit, power balance limit, power loss prevention constraint, and global condition constraint [9-11]. With the development of computer technology and the improvement of communication technology, the research on the energy management strategy of the multienergy ship is more and more in depth. At present, there are mainly fuzzy logic control strategy, baseline control strategy, optimization theory control strategy, logic gate limit control strategy (such as PID control), and modular control strategy. The essence of the fuzzy control strategy is to simulate people's thinking and to design complex tasks with simple strategies. Its main features include that the accuracy of statistical information is very inclusive; it can have a nonlinear controller with fast response speed; and when the system and external parameters change with the height fitting, it can better optimize the performance of each system energy unit and improve the operation cycle and economic performance of the system. But its disadvantage is that the fuzzy rules are based on the operator's experience, which has certain subjective factors and is prone to control distortion $[12,13]$.

The scheduling strategy based on optimization theory is to minimize the fuel and emissions of multienergy SEMS under certain constraints. When the cycle conditions and resistance conditions remain unchanged, a global optimization problem can be obtained, which can be solved by dynamic programming. The modular energy management strategy is a kind of management strategy which divides the complex system into several relatively simple subsystems and selects the appropriate control mode for each subsystem. Reference [14] analyzes the optimal operation of marine electric power system including all electric propulsion and energy storage system. An optimal power management method is proposed to minimize the ship operation cost, limit pollutant emissions, and improve the technical and operational level of the ship power system. Reference [15] proposes a new method to design ship power system and multiagent based power and energy management system, so as to reduce emissions and meet economic and other multiobjectives while minimizing fuel consumption and improving energy efficiency. Reference [16] proposes an energy management system for a fishing vessel power plant with a DC microgrid structure. This kind of EMS has multiobjective functions, such as reducing fuel consumption and pollution emissions and improving the economy. Reference [17] studies the energy storage control strategy of supercapacitor and battery to make the terminal voltage of supercapacitor and battery tend to be balanced and stable in a relatively short time. Reference [18] proposes the energy management system of the electrical system of luxury ships. Starting from the analysis of the current configuration of the electrical system, it puts forward some improvement schemes to reduce the fuel consumption of the diesel driven permanent magnet synchronous generator, so as to reduce the fuel consumption, reduce the pollution emission from related ships, and improve the economy. In [19], an optimal power-flow scheduling of maritime photovoltaic/battery/diesel/cold-ironing hybrid energy systems is proposed to explore solar energy sufficiently and minimize the ship electricity cost. An adaptive multi-context cooperatively coevolving particle swarm optimization (PSO) algorithm is proposed to solve the optimization problem efficiently. In [20], the optimal operation of SIES is modelled as an optimization problem subject to a number of constraints, including emission regulations of ports. Optimal control and model predictive control (MPC) methods are developed to dispatch the power flow when the ship is in port. In addition, the studies on on-land IES can be also used on SIES scheduling problem [21-23]. It can be seen that the intelligent control and optimization methods are widely used in dealing with SIES and on-land IES energy scheduling problems.

From the above works, it is not difficult to find that there are still some problems in the research and application of SIES energy scheduling.

(1) Energy scheduling of SIES is a multiobjective optimization problem, which involves the consideration of economy, environmental protection, reliability, and so on. However, most scholars focus on singleobjective optimization, but a few on multiobjective scheduling. Although some factors are considered during the scheduling process, the evaluation methodology may not be reasonable. Therefore, the scheduling results cannot meet the decision-makers' preference accurately.

(2) The structure of SIES is various, which means different types of ships may have different devices, SEMS, and limits. Thus, the mathematical multiobjective optimization model may change according to different scenarios, which results in that the solution space of the optimization 
problem is complex and changeable. The computation resources of SEMS alone may not meet the requirements of multiobjective energy scheduling.

(3) Comparing with on-land IES, in the actual navigation process, the route of the ship and the task of SIES is often fixed in a certain period. Therefore, the corresponding schemes may have something in common, which can be utilized to obtain knowledge based on on-land high-performance computer system and help the SEMS to get proper schemes online. How to effectively apply the historical data mining results to the calculation of SEMs online scheduling scheme is an urgent problem to be solved, and also the key to realize the green calculation of SEMS.

Motivated by the above discussions, a two-stage multiobjective optimization strategy for SIES economical/environmental scheduling problem (EESP) is proposed. The main novelty and contributions are described as follows:

(1) Considering that there are various types of constraints in real-world SIES-EESPs, some specific constraints handling methods are introduced to deal with different kinds of constraints. With the application of the constraints domination principle and proposed individual repair approach, the algorithm can move to the feasible regions faster.

(2) An establishment method of energy scheduling scheme-base is proposed. The on-land high-performance computer system is utilized to obtain feasible Pareto schemes by big data and the typical solutions are stored. In this way, more computing resources and operation time can be spent to obtain SIESEESPs knowledge.

(3) A short-term multiobjective offline-to-online optimization approach by SEMS is considered, with the application of multiobjective evolutionary algorithm (MOEA) and typical schemes from scheme-bases. Therefore, the rational allocation of computing resources can be realized and the online computing of SIES-EESPs can be more efficient.

The remainder of this paper is organized as follows: the typical SIES-EESP optimization model is built in Section 2. Then, a two-stage multiobjective optimization strategy for SIES-EESP is proposed in Section 3. Thereafter, the case studies are given by comparing the results of the proposed method with other approaches. Finally, the conclusions of this paper are drawn in Section 5.

\section{Typical SIES-EESP Optimization Model}

In this section, a typical SIES is introduced including diesel generators (DGs), energy storage system (ESS), and wind turbine (WT). The mathematical models of output power are presented and the EESP optimization model is built.
2.1. Mathematical Models ofDevices Output. The DGs are the main supplier of marine power. The performance of marine DGs affects the dynamic performance and safety of the ship. In this paper, the structure of diesel engine and generator is simplified, and the fuel consumption is used as the basis to estimate the economic index of the generator set, which can be described as follows:

$$
\begin{aligned}
C_{\text {fuel }}(t) & =a \cdot p(t)^{2}+b \cdot p(t)+c, \\
C_{\text {maintain }}(t) & =m \cdot p(t), \\
C_{\text {start_up }}(t) & = \begin{cases}c, & u(t)=1 \text { and } u(t-1)=0, \\
0, & \text { else, }\end{cases} \\
C_{\text {DG_total }} & =\sum_{t=1}^{T}\left(C_{\text {fuel }}(t)+C_{\text {maintain }}(t)+C_{\text {start_up }}(t)\right),
\end{aligned}
$$

where $C_{\text {fuel }}(t), C_{\text {start_up }}(t)$, and $C_{\text {maintain }}(t)$ are the fuel cost, start-up cost, and the maintenance cost at time $t$, respectively; $c$ is the cold start cost; $u(t)$ is on/off status of the DGs; and $C_{\mathrm{DG} \text { _total }}$ is the total cost of DG in a scheduling period $T$.

In equation (4), the maintenance cost and start-up cost can be calculated by the formulas below:

$$
\begin{aligned}
& C_{\text {maintain }}(t)=P_{\mathrm{DG}}(t) \mathrm{OM}, \\
& C_{\text {start_up }}(t)=\sigma+\delta\left[1-\exp \left(\frac{-T_{\text {off }}(t)}{\tau}\right)\right](1-u(t)),
\end{aligned}
$$

where $\mathrm{OM}$ is the maintenance coefficient; $\sigma$ and $\delta$ are the hot/cold start-up costs of the DG, respectively; $T_{\text {off }}(t)$ is the time the DG has been off; and $\tau$ is the cooling time constant.

The marine WT is installed on the ship. Generally, the wind turbine is installed on the upper deck of the ship to obtain the most wind energy, of which the output power can be obtained by the equation below:

$$
P_{\mathrm{WT}}(t)= \begin{cases}0, & V(t)_{t}<V_{c i}, \\ a_{w} V(t)^{3}+b_{w} V(t)^{2}+c_{w} V(t)+d_{w}, & V_{c i}<V(t)_{t}<V_{r}, \\ P_{r}, & V_{r}<V(t)_{t}<V_{c o}, \\ 0, & V_{t}>V_{c o},\end{cases}
$$

where $P_{r}$ is the rated power of WT; $V_{c i}$ and $V_{c o}$ are the cut-in and cut-out wind speed, respectively; and $V_{r}$ and $V(t)$ are the rated and actual wind speed at time $t$, respectively. $a_{w}, b_{w}, c_{w}$, and $d_{w}$ are the parameters depending on the wind turbine types. The values of all the parameters above can be found elsewhere [24].

The ESS is applied to provide power when the other devices cannot meet the load demand and store electricity when additional power is generated, which can be expressed as [25]

$$
\operatorname{SOC}(t)=\operatorname{SOC}(t-1)+\eta_{\text {char }} P_{\text {char }} \Delta t-\frac{1}{\eta_{\text {dischar }}} P_{\text {dischar }} \Delta t,
$$


where $\operatorname{SOC}(t)$ is the state of charge of ESS; $\eta_{\text {char }}$ and $\eta_{\text {dischar }}$ are the charging and discharging efficiencies; and $\Delta t$ is the time interval.

2.2. Decision Vector. The decision vectors contain the output/input power of the devices and the on/off status of DGs, which can be described as

$$
\mathbf{X}=\left[P_{g}, U_{g}\right]
$$

where $P_{g}$ and $U_{g}$ can be calculated by the following formulas:

$$
\begin{aligned}
& P_{g}=\left[P_{\mathrm{DG}}, P_{\mathrm{ESS}}, P_{\mathrm{WT}}\right], \\
& U_{g}=\left[u_{1}, u_{2}, \ldots, u_{T}\right], \quad u_{k} \in\{0,1\},
\end{aligned}
$$

where $P_{\mathrm{DG}}, P_{\mathrm{ESS}}$, and $P_{\mathrm{WT}}$ are the output/input power vectors of DGs, ESS, and WT in a scheduling period, which can be described by the expressions below:

$$
\begin{aligned}
P_{\mathrm{DG}, i} & =\left[P_{\mathrm{DG}, i}(1), P_{\mathrm{DG}, i}(2), \ldots, P_{\mathrm{DG}, i}(T)\right], \quad i=1,2, \ldots, N_{g}, \\
P_{\mathrm{ESS}} & =\left[P_{\mathrm{ESS}}(1), P_{\mathrm{ESS}}(2), \ldots, P_{\mathrm{ESS}}(T)\right], \\
P_{\mathrm{WT}} & =\left[P_{\mathrm{WT}}(1), P_{\mathrm{WT}}(2), \ldots, P_{\mathrm{WT}}(T)\right],
\end{aligned}
$$

where $P_{\mathrm{DG}, i}(t), P_{\mathrm{ESS}}(t)$, and $P_{\mathrm{WT}}(t)$ are the output power of the $i$-th DG, ESS, and WT, respectively, and $N_{g}$ and $T$ are the number of DGs and the operation period of SIES, respectively.

2.3. Optimization Objectives. There are two economic evaluation indexes for ships, one is the ship income status and the other is the shipping operation cost. In this paper, the operation consumption of SIES is considered as follows:

$$
\text { Cost }=C_{\text {DG_total }}+C_{\text {ESS_total }}+C_{\text {WT_total }} \text {. }
$$

The main air pollution is a diesel generator, which includes COx, Sox, and NOx. The emissions of various pollutants are directly proportional to diesel consumption. This paper uses the ship energy efficiency operation index (EEOI) to evaluate the environmental objectives. The emission value of EEOI includes the emission value during navigation and that of ships berthing in port. The emission subject includes the emission of main and auxiliary engines, boilers, and other equipment, which is also closely related to the sailing distance and cargo carrying capacity, which can be expressed as

$$
\begin{aligned}
\mathrm{EEOI} & =\frac{A}{B}, \\
A & =\sum_{t=1}^{T}\left(\left(c_{2} r_{g}(t)^{2}+c_{1} r_{g}(t)+c_{0}\right) \Delta t\right),
\end{aligned}
$$

$$
B=\sum_{t_{p \in T}}\left(m_{\mathrm{AES}}^{t_{p}} \mathrm{Dist}^{t_{p}}\right)
$$

where $t_{p}$ is the time of berthing, $m_{\mathrm{AES}}^{t_{p}}$ is the cargo load of ships, Dist is the sailing distance; $r_{g}(t)$ is the standard unitary value of the active contribution.

\subsection{Typical Constraints}

2.4.1. Load Balance Constraints. The power output by the devices of SIES should meet the load demand, which can be described as

$$
\sum_{i=1}^{n G} P_{\mathrm{DG}, i}(t) \eta_{g}+P_{\mathrm{ESS}}(t)+P_{\mathrm{WT}}(t)=\frac{P_{l p}(t)}{\eta_{p}}+P_{l s}(t),
$$

where $\eta_{g}$ and $\eta_{p}$ are the efficiency value of DGs and propulsion, respectively. $P_{l p}^{t}$ and $P_{l s}^{t}$ are the load of propulsion and service, respectively.

2.4.2. Rated Power Constraints. The output of DGs and WT should be lower than the rated power, which can be expressed as

$$
r_{g}(t) \leq 1.0
$$

2.4.3. SOC Constraints. The ESS can neither be overcharged nor overused, so the SOC should follow constraint as follows:

$$
\mathrm{SOC}_{\min } \leq \mathrm{SOC}_{t} \leq \mathrm{SOC}_{\max }
$$

2.4.4. Ramp Rate Constraints. The ramp rates of DGs outputs cannot be too large, which can be expressed as

$$
\left|P_{\mathrm{DG}, i}(t)-P_{\mathrm{DG}, i}(t-1)\right| \leq P_{\mathrm{DG}, i, \mathrm{ramp}} .
$$

2.4.5. Minimum On/Off Time Limits. There are minimum on/off time limits for the DGs, which can be expressed as

$$
\begin{aligned}
& \left(T_{\text {on }, i, t-1}-\mathrm{MUT}_{i}\right)\left(u_{i}(t-1)-u_{i}(t)\right) \geq 0, \\
& \left(T_{\text {off }, i, t-1}-\mathrm{MDT}_{i}\right)\left(u_{i}(t)-u_{i}(t-1)\right) \geq 0,
\end{aligned}
$$

where $T_{\text {off }}$ and $T_{\text {on }}$ are the actual on/off time of DGs and MUT and MDT are the minimum on/off time for the DGs.

\section{Two-Stage Multiobjective Optimization Strategy for SIES-EESP}

It can be seen from Section 2 that the SIES contains various types of energy sources and may cost much computation resource when dealing with EESPs by EMS. Therefore, considering the features of SIES discussed in Section 1, a two-stage multiobjective optimization with specific 
constraints handling methods is proposed in this paper to solve the SIES-EESPs.

3.1. Specific Constraints Handling Methods. According to Section 2, there are all kinds of constraints related to SIESEESPs. Some of the constraints are simple but some are not. Therefore, a general constraints handling method may not be effective in finding the feasible regions. In this paper, a comprehensive constraints handling framework is proposed considering specific types of constraints.

3.1.1. Continuous Constraints Handling Strategy. The continuous constraints involved in SIES-EESP are similar to those in other scheduling problems on land, which can be solved effectively by the constraints domination principle (CDP). CDP designed by Deb [26] is an efficient constraint handling approach, which uses the feasibility and the overall constraints violation of solutions to decide the domination levels. To compare the actual engineering constraints fairly, different types of constraints are normalized and summed up first in this paper, which can be expressed as follows:

$$
\begin{aligned}
g_{k, \text { normal }}(\mathbf{x}) & =\frac{g_{k}(\mathbf{x})-g_{k, \text { min }}}{g_{k, \max }-g_{k, \text { min }}} \\
G(\mathbf{x}) & =\sum_{k=1}^{k} w_{k} g_{k, \text { normal }}(\mathbf{x}),
\end{aligned}
$$

where $g_{k \text {,normal }}$ and $g_{k}$ are the normalized violation value and actual value of the k-th type of constraint, respectively; $g_{k \text {, min }}$ and $g_{k, \max }$ are the minimum and maximum violation value of the $\mathrm{k}$-th type of constraint in the population, respectively; and $G$ is the overall constraints violation value.

\subsubsection{Discrete Constraints Handling Strategy. It can be seen} from equation (11) that there are discrete variables $(0 / 1)$ in the decision variable vector, which represent the on/off statuses at those time points. In the MOEA process, these discrete variables cannot be optimized gradually during evolution, and it is always difficult to measure the gap between the infeasible discrete variable vector and the feasible region. Therefore, in a practical SIES-EESP, it may not be efficient to handle the constraints related to the discrete variable vector (such as the on/off time limits).

To avoid the violations of this constraint when using MOEA, this paper proposes an individual repair approach (IRA) based on variables separation and recombination.

The repair process of the decision vector is actually to modify the infeasible subvector $u_{i}$, which can be described as the following steps:

(i) Step 1: For the status variable vector $\mathbf{u}_{i}=\left[u_{i}(1), u_{i}(2), \ldots, u_{i}(T)\right]$ of the i-th DG, decompose it into several subvectors, where there are only ones or zeros as elements. Separate the subvectors into two sets by the elements, in which Set $U$ only has subvectors composed of ones and Set D only has subvectors composed of zeros. Then, the numbers of the subvector dimensions will be composed to a new vector in each set, which can be described as $x_{U}=\left[x_{U, 1}, x_{U, 2}, \ldots, x_{U, u}\right]$ and $x_{D}=\left[x_{D, 1}, x_{D, 2}, \ldots, x_{D, d}\right]$, respectively. Then, the elements in $x_{U}$ and $x_{D}$ will be recombined into a new vector $\mathbf{x}_{\mathrm{UD}}$, according to the order of elements $\mathbf{u}_{i}$.

(ii) Step 2: If $u_{k}(1)=1$, go to Step 3; if not, move to Step 4.

(iii) Step 3: From the first nonzero element of $\mathbf{x}_{\mathrm{UD}}$, check all of the nonzero elements from left to right. For the nonzero elements in the odd positions, identify whether they meet the minimum on-time limits, while for those in the even positions, determine whether they meet the minimum off-time limits. Suppose that there is a nonzero element in the odd position (let us say $x_{U D, j}$ ) which does not meet the constraint. In another word, if $\mathrm{MUT}_{k}-x_{\mathrm{UD}, j}=$ $\Delta U_{j}>0$, the modification procedure starts, of which the pseudocode is presented in Algorithm 1. After the modification procedures, the new vector $\mathbf{x}_{\mathrm{UD}}{ }^{\prime}$ is obtained. Then, go to Step 5 .

(iv) Step 4: This step is similar to Step 3. The only difference is that for the nonzero elements in the odd positions, identify whether they meet the minimum off-time limits, and for those in the even positions, determine whether they meet the minimum on-time limits. Algorithm 1 still applies to the violations. Then, the repair process moves to Step 5 .

(v) Step 5: remove the zero elements of vector $\mathbf{x}_{\mathrm{UD}}{ }^{\prime}$ and keep the nonzero elements in the original order, which form a new vector $\mathbf{x}_{\mathrm{UD} \text {,repair }}$. The elements of vector $\mathbf{x}_{\mathrm{UD} \text {,repair }}$ represent the amount of the on/off hours. The on and off statuses of the DGs occur alternately, and whether the first status is on or off is related to value of $u_{i}(1)$.

(vi) Step 6: establish the feasible status variable vector $u_{i}^{\prime}$ based on $\mathbf{x}_{\mathrm{UD}}^{\prime}$ and $u_{i}(1)$.

It is obvious from the repair process that the relationship between the status variable vector $\mathbf{u}_{i}$ generated randomly in the T-dimensional solution space $Q_{T}$ and the obtained feasible status variable vector $u_{i}^{\prime}$ is a many-to-one mapping, which means that, by this proposed method any infeasible status variable vectors can be transferred into a certain vector in the feasible region, and no feasible vectors would be lost. To guarantee the effectiveness of the optimization process, the replacement ratio is set $15 \%$ according to [27].

3.2. Offline-to-Online Multiobjective Optimization Strategy. The specific constraints handling methods can handle different types of constraints during the evolutionary process on actual SIES-EESPs, which can help MOEA to find feasible solutions efficiently. However, it is evident that the constraints handling process may require excessive computational resources and reduce the efficiency of EMS in dealing with energy dispatch problems. Therefore, an offline-to-online multiobjective optimization strategy is 


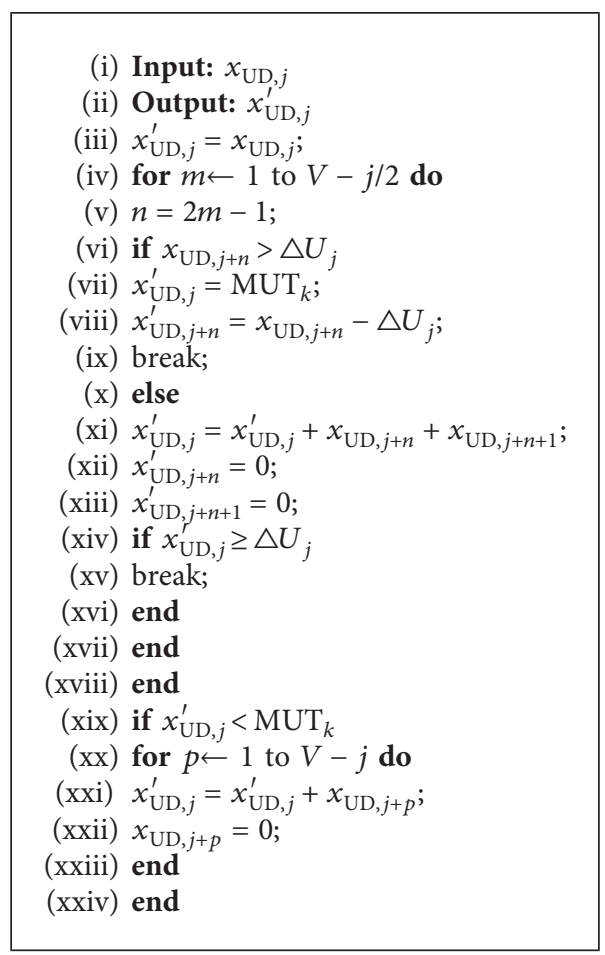

Algorithm 1: Modification procedure.

proposed to transfer some of the computing tasks from SEMS to offline computers on land.

\subsubsection{Establishment of Energy Scheduling Scheme-Base Using} Big Data. Since the courses and tasks of inland cargo ships are similar in the same months of a year, of which the scheduling schemes may not have much difference, it is possible to use the historical experiences to guide the dayahead economical/environmental scheduling optimization. By using the big data of the typical curves of load demands, the wind speed of specific regions can be fitted out and the applications of all kinds of energy resources can be derived. On the other hand, the establishment of the energy scheduling scheme-base using big data can be realized by a high-performance computer system with more computing resources on land $[28,29]$. Therefore, the scheme-base for a specific inland cargo ship can be obtained including all the economical/environmental scheduling solutions of a typical year by big data. Each scheduling task is formed as a SIESEESP with fitted curves of load and wind speed. And the MOEA is applied with the proposed specific constraints handling methods to search for the typical Pareto schemes. Since there are no time and computing resources limits, this process can be realized on the high-performance computer system and stored in the mass storage devices on land.

The typical Pareto schemes would be stored in schemebase for SIES-EESP, which contains two subbases, namely, Scheme-base A and Scheme-base B, which store the feasible Pareto SIES-EESP schemes meeting all the constraints and only the discrete constraints, respectively. By introducing the high-performance computers on land with large memory space and unlimited operation time, the obtained
Pareto solutions can be more close to the true Pareto frontiers and the typical schemes can ensure the low cost and emission under various types of working conditions of a year.

3.2.2. Short-Term Multiobjective Offline-to-Online Optimization by SEMS. Although the economical/environmental scheduling solutions in the scheme-base can be applied in typical working conditions, it is clear that, in practice, the load may change according to the actual requirement, and the environment can also be different so that the output of renewable energy sources would not follow the typical schemes. Therefore, the short-term dispatch scheme is still needed by the SEMS based on its own conditions. To reduce the operation time of SEMS when dealing with SIES-EESPs, a novel multiobjective offline-to-online optimization strategy is proposed based on the energy scheduling scheme-base. The procedure can be described as follows:

(i)Step 1: Select the Pareto energy scheduling scheme sets of the typical SIES-EESP which has the highest fitting degree with the short-term forecast curves of load demand and wind speed.

(ii)Step 2: Choose a kind of MOEA as the optimization tool and use CDP and IRA as the constraints handling methods.

(iii)Step 3: Use the typical solutions in Scheme-base A to form the initial population.

(iv)Step 4: Run MOEA to find the Pareto solutions of the actual SIES-EESP. During the evolutionary process, check the proportion $\alpha$ of the infeasible solutions. If $\alpha>\alpha_{0}$, move to Step 5; otherwise, go to Step 6 . 
(v)Step 5: Replace the infeasible solutions with the typical solutions in Scheme-base B in the current population, so that the algorithm can move to the feasible regions corresponding to the discrete constraints efficiently.

(vi)Step 6: Output the Pareto solutions.

It can be seen from the above that comparing with the traditional optimum dispatching methods, which utilize the forecast data and solve the SIES-EESPs in one operation, the proposed two-stage offline-to-online multiobjective optimization strategy can utilize the big data to find the knowledge of the SIES-EESPs by offline computers on land so that the reference typical solutions are derived and stored. When a new actual SIES-EESP needs to be solved, the SEMS can find the Pareto solutions with the help of the most relevant reference schemes from the solution-base. In this way, the online computing resources can be saved and the computing efficiency is improved.

The flowchart of the short-term multiobjective offlineto-online optimization by SEMS is shown in Figure 1.

\section{Simulation Results and Discussions}

4.1. Data and Parameter Settings. In this section, two actual SIES-EESP cases are introduced considering different combinations of energy sources. In Case 1, there are only two DGs and one ESS, and, in Case 2, a WT is introduced as the renewable energy source. The parameter settings of ESS and WT can be found in Table 1. Other parameter settings can be found in $[24,30]$. The fitted curves by big data and the actual curves of the load demand and wind speed are shown in Figures 2(a) and 2(b).

Besides, in this paper, the nondominated sorting genetic algorithm (NSGA-II) is utilized as the core optimization algorithm, which is an efficient MOEA in solving all kinds of multiobjective optimization problems. The parameter settings can be found in [26]. The maximum generation is set as 100000 when searching for the typical Pareto schemes. In addition, to study the performance of the proposed strategy, four methods are utilized. Method 1 and Method 2 use the proposed offline-to-online optimization strategy, of which the difference is that only Method 1 uses the specific constraints handling methods to deal with the constraints while Method 2 only uses CDP. Method 3 is the original NSGAII with CDP, but in Method 4, the punishment function method is introduced, which can be found in [25]. The maximum generation is 2000 for Methods 1 and 2 and 10000 for Methods 3 and Method 4, respectively.

4.2. Case Studies. The average values of best cost and emission for the two cases by the four methods can be found in Table 2. It can be seen from Table 2 that the results by Methods 1 and 2 are similar in the two cases, and the results in Case 2 are better, which indicates that by introducing the wind turbine, renewable energy can be made full use of and the cost and pollutions can be reduced. The results also show that, by utilizing the typical solutions in the scheme-base as reference individuals, the algorithm can find better solutions so that the distribution of the Pareto frontiers can be guaranteed. On the contrary, if NSGAII is used directly without any prior knowledge of the related SIES-EESP, the algorithm may trap in local optimum and cannot find satisfying nondominate solutions for the decision-makers. The results of Methods 3 and 4 also show that the CDP method is better than PFM in dealing with SIES-EESP cases.

To further study the performance of the proposed two-stage multiobjective optimization strategy, the population of the last generation, the feasible solutions, and the Pareto frontiers obtained by the four methods are presented in Figures 3-5. Also, the average numbers of feasible solutions are shown in Table 3.

It can be seen from Figures 3(a) and 5 that almost all of the solutions by Method 1 are nondominate comparing with those by other methods. Besides, nearly 48 solutions are feasible ones, and the solutions distribute evenly on the Pareto frontier, which can provide more trade-off schemes for the decision-makers. As for Method 2, most of the solutions are dominated by those found by Method 1 but are much better than those using Methods 3 and 4. The numbers of obtained feasible solutions are also lower than those by Method 1, most of which are located in a smaller region. The results by Methods 3 and 4 are the worst in Cases 1 and 2, which have higher cost and emission values comparing with those by Methods 1 and 2. It can be seen from Figure 3 and that both Methods 3 and 4 trap in local optimum and all the solutions obtained are dominated by other methods. Moreover, according to Figures 4(a) and 4(b), only about 28 and 15 solutions in the two cases are feasible for Method 3, and about 22 and 10 feasible ones are obtained by Method 4 . Based on the results in Figure 5, no more than 6 solutions are nondominate in the population for the last two methods, which means they cannot provide enough trade-off schemes for the decision-makers.

The results in Cases 1 and 2 imply that, with the applications of energy scheduling scheme-base, the algorithm can jump out of the infeasible regions and find the feasible regions efficiently. Therefore, more computing resources can be utilized to search for the Pareto solutions, so that the convergence and diversity of the population can be guaranteed. On the other hand, the introduction of specific constraints handling methods, especially the proposed IRA for discrete constraints, helps the algorithm to deal with all kinds of constraints effectively, so more feasible Pareto solutions can be obtained. In addition, the results by Methods 3 and 4 indicate that CDP is efficient in dealing with common constraints and is necessary for the shortterm multiobjective offline-to-online optimization.

To evaluate the Pareto frontiers obtained by the four methods quantitatively, the hypervolume method is introduced. The evaluation method based on hypervolume value was proposed by Zitzler et al. [31], which utilizes the volume of hypercube surrounded by the Pareto solutions and reference point in the search space to evaluate the quality of Pareto frontiers obtained. The average values and standard deviations of hypervolume values by the four methods are shown in Table 4. It can be seen from 


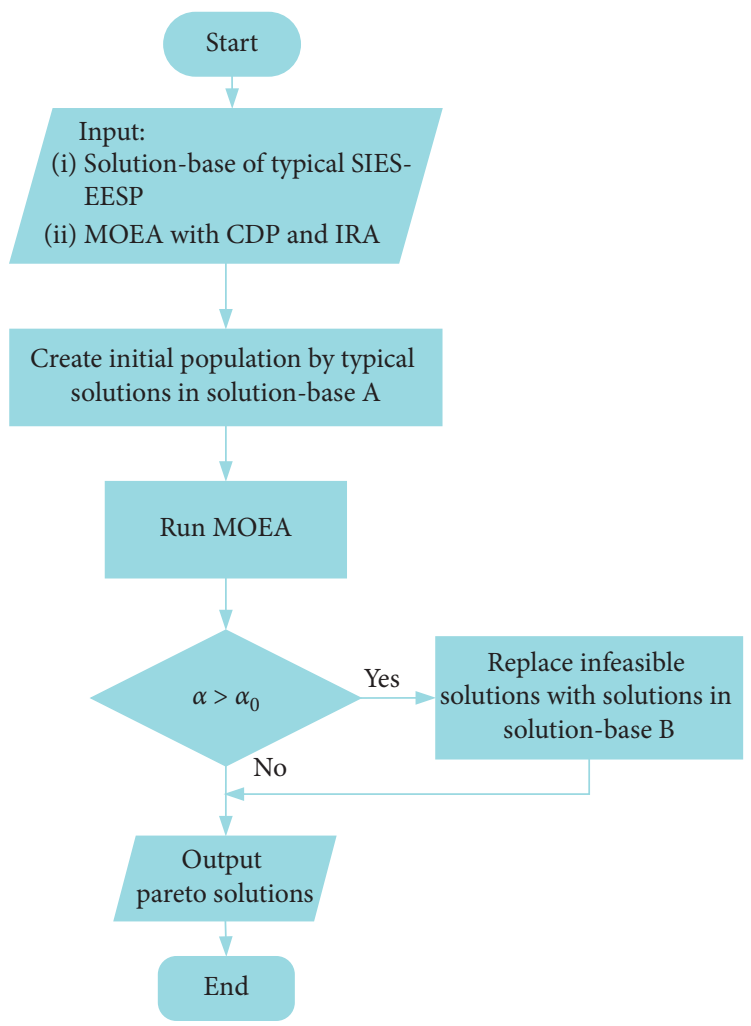

FIGURE 1: Flowchart of short-term multiobjective offline-to-online optimization by SEMS.

TABLE 1: Parameter settings of ESS.

\begin{tabular}{lc}
\hline Initial cost $(\mathrm{m} . \mathrm{u} . / \mathrm{kW})$ & 567 \\
Replacement cost $(\mathrm{m} . \mathrm{u} . / \mathrm{kW})$ & 467.9 \\
Maintenance cost $(\mathrm{kW} \cdot \mathrm{h} \cdot \mathrm{y})$ & 2.67 \\
Charging efficiency & 0.86 \\
Discharge efficiency $_{\text {SOC }_{\max }}$ & 0.86 \\
SOC $_{\min }$ & 0.9 \\
Initial SOC $_{\text {Service life }(\mathrm{yr})}$ & 0.3 \\
\hline
\end{tabular}

Table 4 that Method 1 obtains the highest hypervolume values in both cases (0.7051 and 0.8014). The hypervolume values by Method 2 are lower but the difference is small. However, Method 3 only gets 0.1648 and 0.0737 on hypervolume values, which are far lower than the other two. This demonstrates that if the original NSGAII is utilized to solve the actual SIES-EESP, it may not get satisfied Pareto solutions with limited computing resources and operation time, so the goals of cost and emission reduction cannot be achieved. The hypervolume values by Method 4 are the lowest, which means the commonly used punishment function methods are not suitable for actual SIES-EESPs. However, with the utilization of energy scheduling scheme-base and proposed constraints handling method, the feasible regions can be found efficiently and the Pareto sets can be obtained with better convergence, spread, and distribution by less computing resources and operation time.

As for the standard deviations, it can be seen from Table 4 that Method 1 gets the lowest levels, which means that the proposed strategy is more reliable in searching for Pareto solutions on the SIES-EESPs. This is necessary in the real world since the online dispatching requires the SEMS to obtain the Pareto schemes in a short time, which does not allow the algorithm to run many times. Thus, a reliable optimization tool is needed to ensure a reliable/economical/environmental dispatching scheme.

The average CPU time by the four methods is shown in Table 5. Since Methods 3 and 4 take iterative 10000 times, the CPU time is much larger than the other two. According to the results above, it is evident that, by using the typical solutions from the energy scheduling schemebase, the SEMS can get more feasible and nondominate solutions within less operation time, which can save more computing resources. In addition, it can be seen that Method 1 takes a little more time than Method 2 because of the introduction of specific constraints handling 


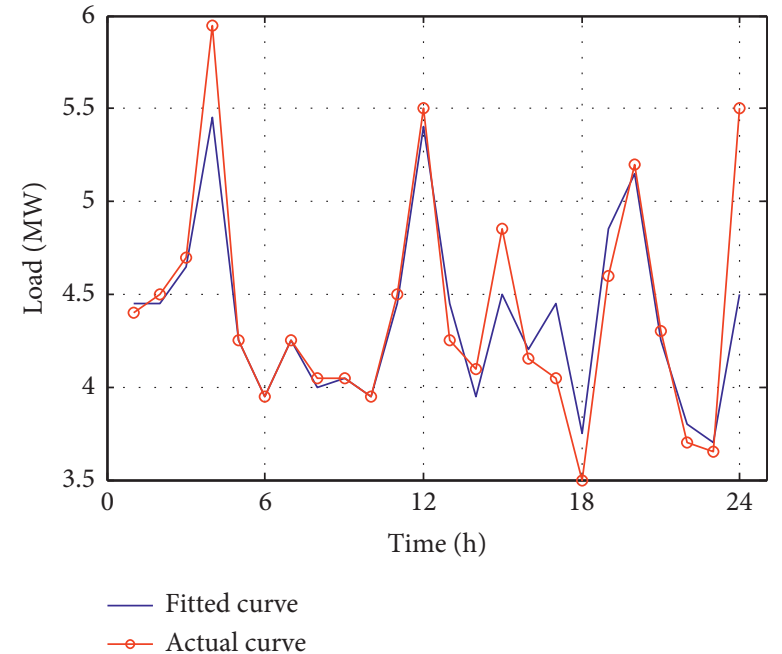

(a)

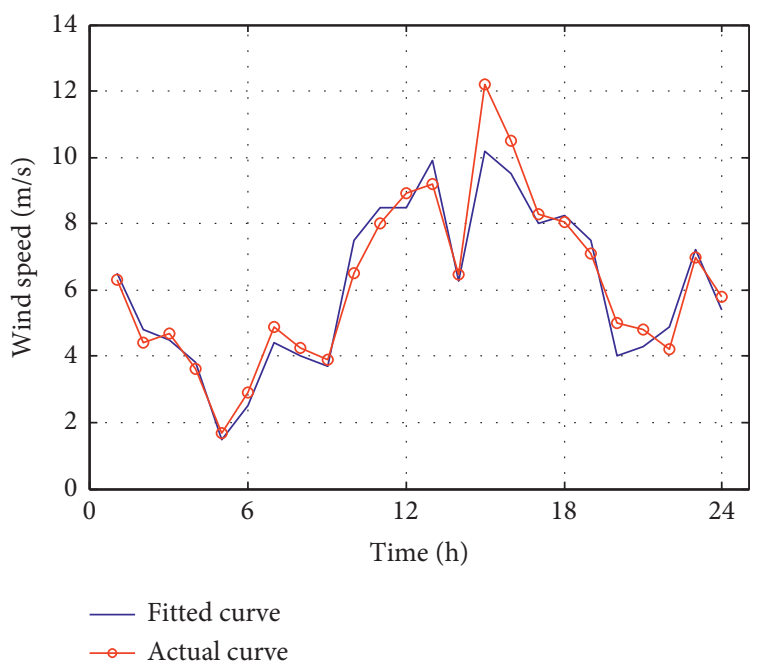

(b)

Figure 2: Fitted curves by big data and the actual curves of the load demand and wind speed. (a) Load demand. (b) Wind speed.

TABle 2: Average values of best cost and emission by the four methods.

\begin{tabular}{|c|c|c|c|c|c|c|c|c|}
\hline \multirow[b]{2}{*}{ Cases } & \multicolumn{2}{|c|}{ Method 1} & \multicolumn{2}{|r|}{ Method 2} & \multicolumn{2}{|r|}{ Method 3} & \multicolumn{2}{|r|}{ Method 4} \\
\hline & $\begin{array}{l}\text { Cost } \\
\text { (m.u.) }\end{array}$ & $\begin{array}{c}\text { Emission } \\
\left(10^{4} \text { u. } \mathrm{CO}_{2}\right)\end{array}$ & Cost (m.u.) & Emission $\left(10^{4}\right.$ u. $\left.\mathrm{CO}_{2}\right)$ & Cost (m.u.) & Emission $\left(10^{4}\right.$ u. $\left.\mathrm{CO}_{2}\right)$ & Cost (m.u.) & Emission $\left(10^{4}\right.$ u. $\left.\mathrm{CO}_{2}\right)$ \\
\hline Case 1 & 7.8254 & 4.4166 & 7.9610 & 4.4621 & 8.6542 & 4.8458 & 8.6721 & 4.8593 \\
\hline Case 2 & 7.0542 & 4.2985 & 7.2544 & 4.4203 & 7.8461 & 4.5319 & 7.8836 & 4.5546 \\
\hline
\end{tabular}

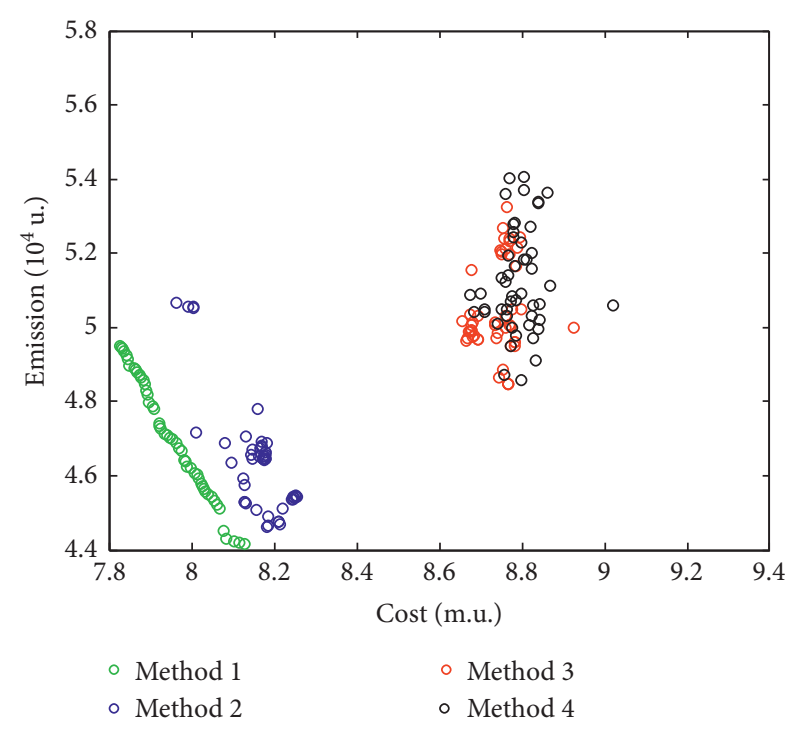

(a)

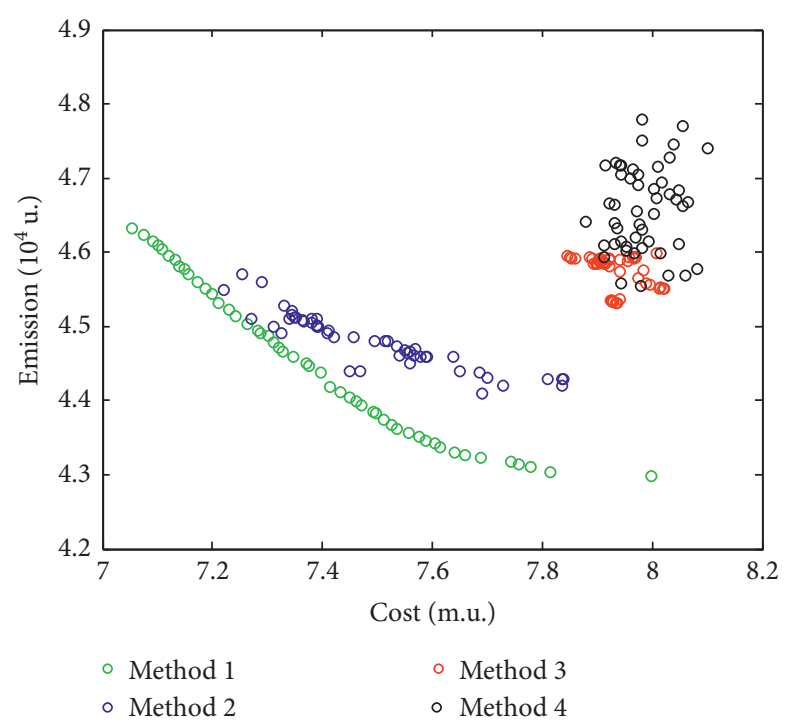

(b)

Figure 3: Populations of the last generation by the four methods. (a) Case 1. (b) Case 2.

methods, but the difference is not large (only about $5 \mathrm{~s}-7 \mathrm{~s})$. On the other hand, the number of feasible solutions and the hypervolume values by Method 1 are better based on the above results. All in all, the proposed Method 1 is a proper optimization tool for the SEMS in dealing with the SIES-EESPs. 


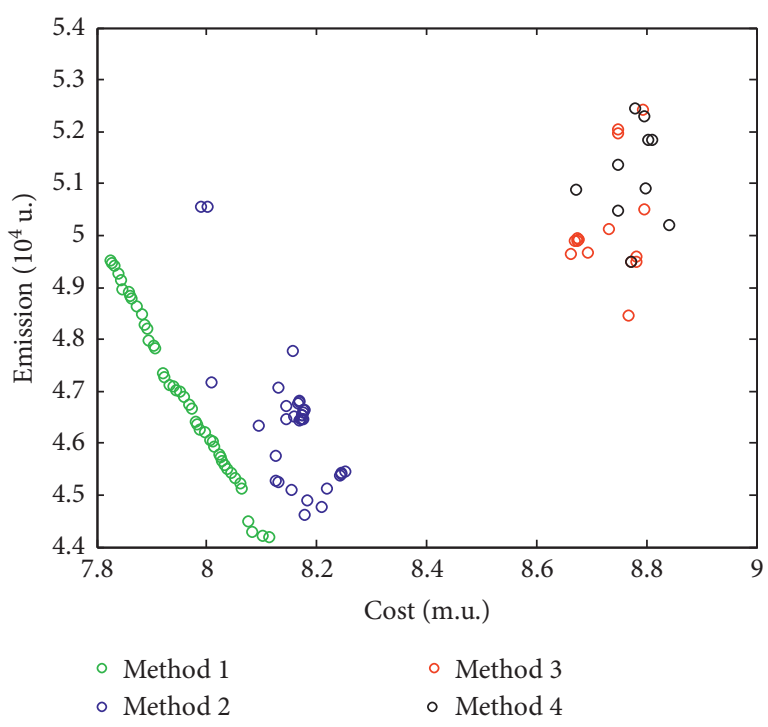

(a)

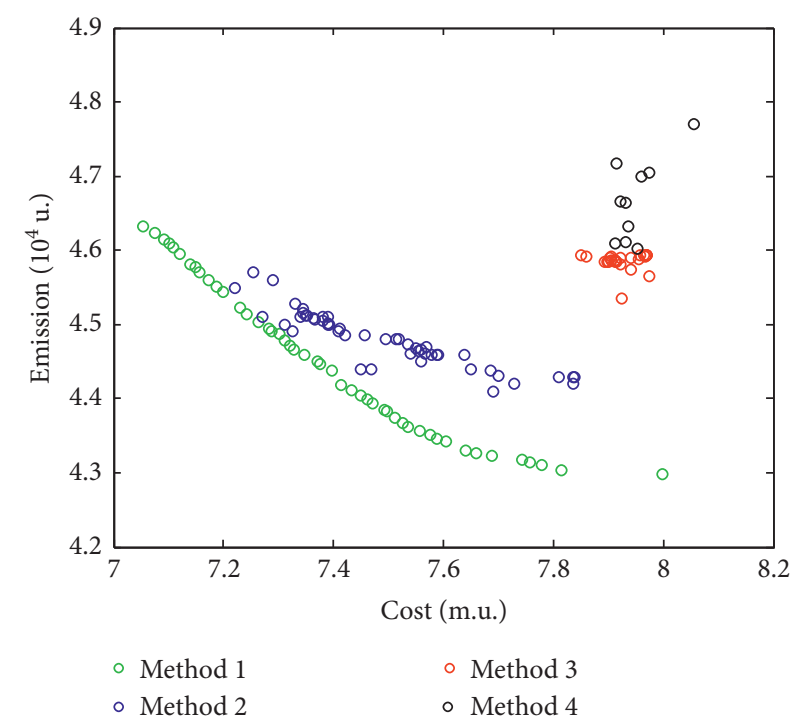

(b)

FIgUre 4: Feasible solutions by the four methods. (a) Case 1. (b) Case 2.

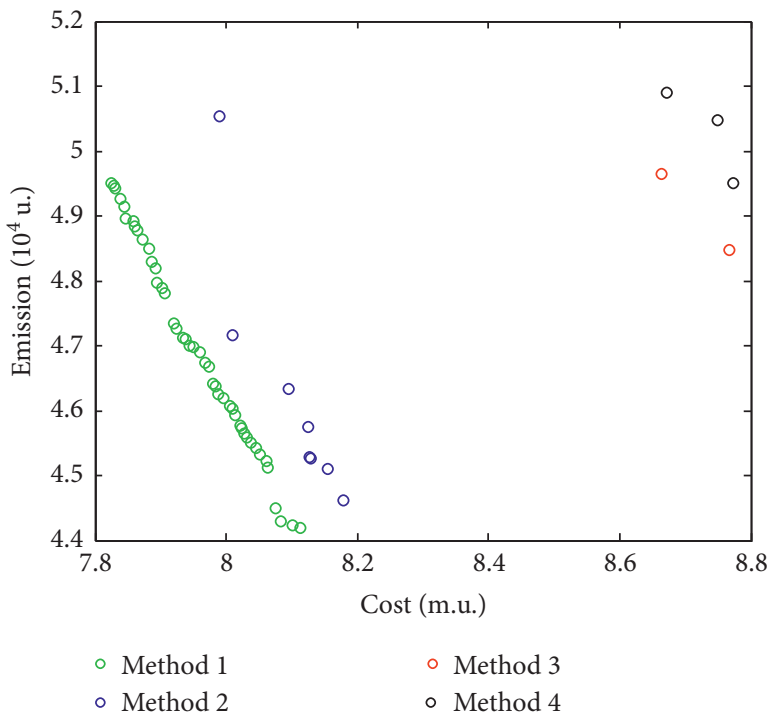

(a)

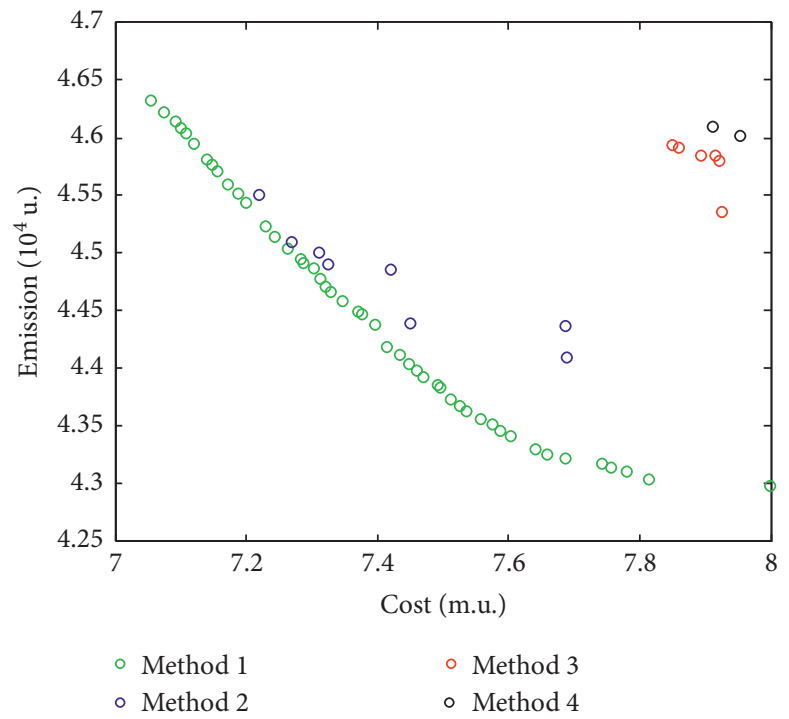

(b)

Figure 5: Pareto frontiers obtained by the four methods. (a) Case 1. (b) Case 2.

TABle 3: Average number of feasible solutions by the four methods.

\begin{tabular}{lcccc}
\hline Cases & Method 1 & Method 2 & Method 3 & Method 4 \\
\hline Case 1 & 47.8 & 42.0 & 28.2 & 21.3 \\
Case 2 & 45.3 & 32.5 & 15.1 & 9.8 \\
\hline
\end{tabular}

TABLE 4: Average values and standard deviations of hypervolume values by the four methods.

\begin{tabular}{lcccccccc}
\hline \multirow{2}{*}{ Cases } & \multicolumn{2}{c}{ Method 1 } & \multicolumn{2}{c}{ Method 2 } & \multicolumn{2}{c}{ Method 3 } & \multicolumn{2}{c}{ Method 4 } \\
& H-values & Standard deviations & H-values & Standard deviations & H-values & Standard deviations & H-values & Standard deviations \\
\hline Case 1 & 0.7051 & $5.2314 e-03$ & 0.6241 & $9.9878 e-03$ & 0.1648 & $3.2148 e-02$ & 0.1135 & $7.2615 e-02$ \\
Case 2 & 0.8014 & $7.2457 e-03$ & 0.6987 & $1.5414 e-02$ & 0.0737 & $7.0325 e-02$ & 0.0341 & $7.9984 e-02$ \\
\hline
\end{tabular}


TABle 5: Average CPU time(s) by the four methods.

\begin{tabular}{lcccc}
\hline Cases & Method 1 & Method 2 & Method 3 & Method 4 \\
\hline Case 1 & 36.9827 & 31.2548 & 250.2489 & 312.5132 \\
Case 2 & 41.0065 & 34.0124 & 259.3257 & 333.2147 \\
\hline
\end{tabular}

\section{Conclusions}

This paper proposes a two-stage offline-to-online multiobjective optimization strategy for SIES-EESP, by which the SIES-EESP schemes are derived by using the big data offline to form the prior knowledge and the MOEAs to search for feasible Pareto solutions online. Before the application of intelligent computing, the hybrid constraints handling strategies are designed considering both continuous constraints and discrete constraints. Then, the energy scheduling scheme-base is established using big data, so that the typical SIES-EESP schemes can be obtained and stored offline by high-performance computer systems. On the other hand, the typical Pareto solutions in the scheme-base are applied to the actual SIES-EESPs online corresponding to similar load demands and environment conditions, which are used as reference vectors to guide the algorithm to converge to the feasible regions. The results show that the proposed method can obtain enough feasible solutions and get well-distributed Pareto sets with better convergence performance. Moreover, the operation time can be reduced evidently with less computing resources, comparing with the current optimization studies. By the two-stage multiobjective optimization strategy, the rational allocation of computing resources and the advantage of SIES big data and high-performance computer systems on land can be realized. Meanwhile, the EMS only needs to select the most relevant reference schemes and uses them to find the Pareto solutions on actual SIES-EESPs. In this way, part of the computing task is transferred to the offline computers on land, so that the online computing resources can be saved. It should be noted that this paper only considers a typical SIES-EESP optimization model and divide the constraints into two types (general ones and special ones) to establish the scheme-bases. Further studies are needed on the following aspects:

(1) More SIES-EESP optimization models can be studied based on the needs in the real world so that the adaptability of the proposed method can be tested.

(2) More types of constraints can be derived to describe the SIES-EESP more accurately, and the schemebases can be established based on more kinds of rules. In this way, the selected typical solutions during the online computing process by SEMS can help MOEA to reach feasible regions more efficiently.

(3) More kinds of MOEAs can be utilized in the proposed optimization strategy so that a proper MOEA can be selected when dealing with a specific actual SIES-EESP.

\section{Data Availability}

The prior studies and data are cited at relevant places within the text as references $[24-26,30]$.

\section{Conflicts of Interest}

The authors declare that they have no conflicts of interest.

\section{Acknowledgments}

This work was supported by the National Natural Science Foundation of China (Grants nos. 51909199 and 51709215), the Green Intelligent Inland Ship Innovation Programme, by projects from Key Lab of Marine Power Engineering and Tech. Authorized by MOT (KLMPET2019-03 and KLMPET2019-02), and the Opening Foundation of Key Laboratory of Information Security of Zhejiang Province (Grant no. KF201912).

\section{References}

[1] W. Zhou and X. Xiaohua, "Tariff-driven demand side management of green ship," Solar Energy, vol. 170, pp. 991-1000, 2018.

[2] L. Bilgili and U. B. Celebi, "Developing a new green ship approach for flue gas emission estimation of bulk carriers," Measurement, vol. 120, pp. 121-127, 2018.

[3] J. Lampe, E. Rüde, Y. Papadopoulos, and S. Kabir, "Modelbased assessment of energy-efficiency, dependability, and cost-effectiveness of waste heat recovery systems onboard ship," Ocean Engineering, vol. 157, pp. 234-250, 2018.

[4] Y. Hongsheng, W. Liyang, and Y. Jianxing, "The environmental impact analysis of hazardous materials and the development of green technology in the shipbreaking process," Ocean Engineering, vol. 161, pp. 187-194, 2018.

[5] Z. Li, Y. Xu, L. Wu, and X. Zheng, "A risk-averse adaptively stochastic method for multi-energy ship operation under diverse uncertainties," IEEE Transactions on Power Systems, 2020.

[6] Z. Li, Y. Xu, S. Fang et al., "Robust coordination of A hybrid AC/DC multi-energy ship microgrid with flexible voyage and thermal loads," IEEE Transactions on Smart Grid, vol. 99, p. 1, 2020.

[7] Z. Li, Y. Xu, S. Fang, Y. Wang, and X. Zheng, "Multiobjective coordinated energy dispatch and voyage scheduling for a multienergy ship microgrid," IEEE Transactions on Industry Applications, vol. 56, no. 2, pp. 989-999, 2020.

[8] C. Zhang, D. Zhang, M. Zhang et al., "Data-driven ship energy efficiency analysis and optimization model for route planning in ice-covered arctic waters," Ocean Engineering, vol. 186, Article ID 106071, 2019.

[9] E. H. Trinklein, G. G. Parker, and T. J. Mccoy, "Modeling, optimization, and control of ship energy systems using exergy methods," Energy, vol. 191, pp. 1-8, 2020.

[10] S. Fang and Y. Xu, "Multi-objective robust energy management for all-electric shipboard microgrid under uncertain wind and wave," International Journal of Electrical Power and Energy Systems, vol. 117, pp. 1-11, 2020.

[11] N. Vahabzad, M. Jadidbonab, B. Mohammadiivatloo et al., "Energy management strategy for a short-route hybrid cruise ship: an IGDT-based approach," IET Renewable Power Generation, vol. 14, no. 10, 2020.

[12] Q. Zhang, Z. Ding, and M. Zhang, "Adaptive self-regulation PID control of course-keeping for ships," Polish Maritime Research, vol. 27, no. 1, pp. 39-45, 2020.

[13] A. Ouroua, L. Domaschk, and J. H. Beno, "Electric ship power system integration analyses through modeling and 
simulation," in Proceedings of the Electric Ship Technologies Symposium, IEEE, Paris, France, October 2005.

[14] F. D. Kanellos, "Optimal power management with GHG emissions limitation in all-electric ship power systems comprising energy storage systems," IEEE Transactions on Power Systems, vol. 29, no. 1, pp. 330-339, 2013.

[15] D. Tang, X. Yan, Y. Yuan et al., "Multi-agent based power and energy management system for hybrid ships," in Proceedings of the 2015 International Conference on Renewable Energy Research and Applications (ICRERA), pp. 383-387, IEEE, Palermo, Italy, November 2015.

[16] A. Accetta and M. Pucci, "A first approach for the energy management system in DC micro-grids with integrated RES of smart ships," in Proceedings of the 2017 IEEE Energy Conversion Congress and Exposition (ECCE), pp. 550-557, IEEE, Cincinnati, OH, USA, October 2017.

[17] Z. Jingnan and Z. Ying, "Control strategy of hybrid energy storage system in ship electric propulsion," in Proceedings of the 2018 IEEE International Conference on Mechatronics and Automation (ICMA), pp. 1026-1030, IEEE, Changchun, China, August 2018.

[18] A. Accetta and M. Pucci, "Energy management system in DC micro-grids of smart ships: main gen-set fuel consumption minimization and fault compensation," IEEE Transactions on Industry Applications, vol. 55, no. 3, pp. 3097-3113, 2019.

[19] R. Tang, X. Li, J. Lai et al., "A novel optimal energy-management strategy for a maritime hybrid energy system based on large-scale global optimization," Applied Energy, vol. 228, pp. 254-264, 2018.

[20] R. Tang, Z. Wu, X. Li et al., "Optimal operation of photovoltaic/battery/diesel/cold-ironing hybrid energy system for maritime application,” Energy, vol. 162, pp. 697-714, 2018.

[21] L. Jinglu, W. Anna, Q. Yanhua et al., "Coordinated operation of multi-integrated energy system based on linear weighted sum and grasshopper optimization algorithm," IEEE Access, vol. 6 , p. 1, 2018

[22] A. Chaouachi, R. M. Kamel, R. Andoulsi, and K. Nagasaka, "Multiobjective intelligent energy management for a microgrid," IEEE Transactions on Industrial Electronics, vol. 60, no. 4, pp. 1688-1699, 2013.

[23] A. B. Ani, H. A. Ebrahim, and M. J. Azarhoosh, "Simulation and multi-objective optimization of a trickle-bed reactor for diesel hydrotreating by a heterogeneous model using nondominated sorting genetic algorithm II," Energy and Fuels, vol. 29, pp. 3041-3051, 2015.

[24] X. Li and Y. Fang, "Dynamic environmental/economic scheduling for microgrid using improved MOEA/D-M2M," Mathematical Problems in Engineering, vol. 2016, Article ID 2167153, 14 pages, 2016.

[25] X. Li, J. Lai, and R. Tang, "A hybrid constraints handling strategy for multiconstrained multiobjective optimization problem of microgrid economical/environmental dispatch," Complexity, vol. 2017, Article ID 6249432, 12 pages, 2017.

[26] K. Deb, A. Pratap, S. Agarwal, and T. Meyarivan, "A fast and elitist multiobjective genetic algorithm: NSGA-II," IEEE Transactions on Evolutionary Computation, vol. 6, no. 2, pp. 182-197, 2002.

[27] L. Y. Tseng and C. Chen, "Multiple trajectory search for single objective constrained real-parameter optimization problems," in Proceedings of the IEEE Congress on Evolutionary Computation, pp. 1-7, IEEE, Barcelona, Spain, July 2010.

[28] J. Lai, X. Lu, X. Yu, and A. Monti, "Stochastic distributed secondary control for ac microgrids via event-triggered communication," IEEE Transactions on Smart Grid, vol. 11, no. 4, pp. 2746-2759, 2020.

[29] J. Lai, X. Lu, X. Yu, and A. Monti, "Cluster-oriented distributed cooperative control for multiple ac microgrids," IEEE Transactions on Industrial Informatics, vol. 15, no. 11, pp. 5906-5918, 2019.

[30] X. Chen, Q. Wei, and X. Li, Research on Multiobjective Optimization Strategy of Economic/Environmental Energy Management for Multi-Energy Ship Based on MOEA/D, BioInspired Computing: Theories and Applications, pp. 135-146, 2020.

[31] E. Zitzler and L. Thiele, "Multiobjective evolutionary algorithms: a comparative case study and the strength Pareto approach," IEEE Transactions on Evolutionary Computation, vol. 3, no. 4, pp. 257-271, 1999. 